\title{
Optical Assembly and Alignment for the National Ignition Facility Project
}

\author{
P. A. Hurst, E. L. Grasz, H. Wong, \\ E. H. Schmitt, and M. R. Simmons
}

This paper was prepared for and presented at the SPIE International Symposium on High-Power Lasers and Applications San Jose, California

January 24-30, 1998

December 23, 1997

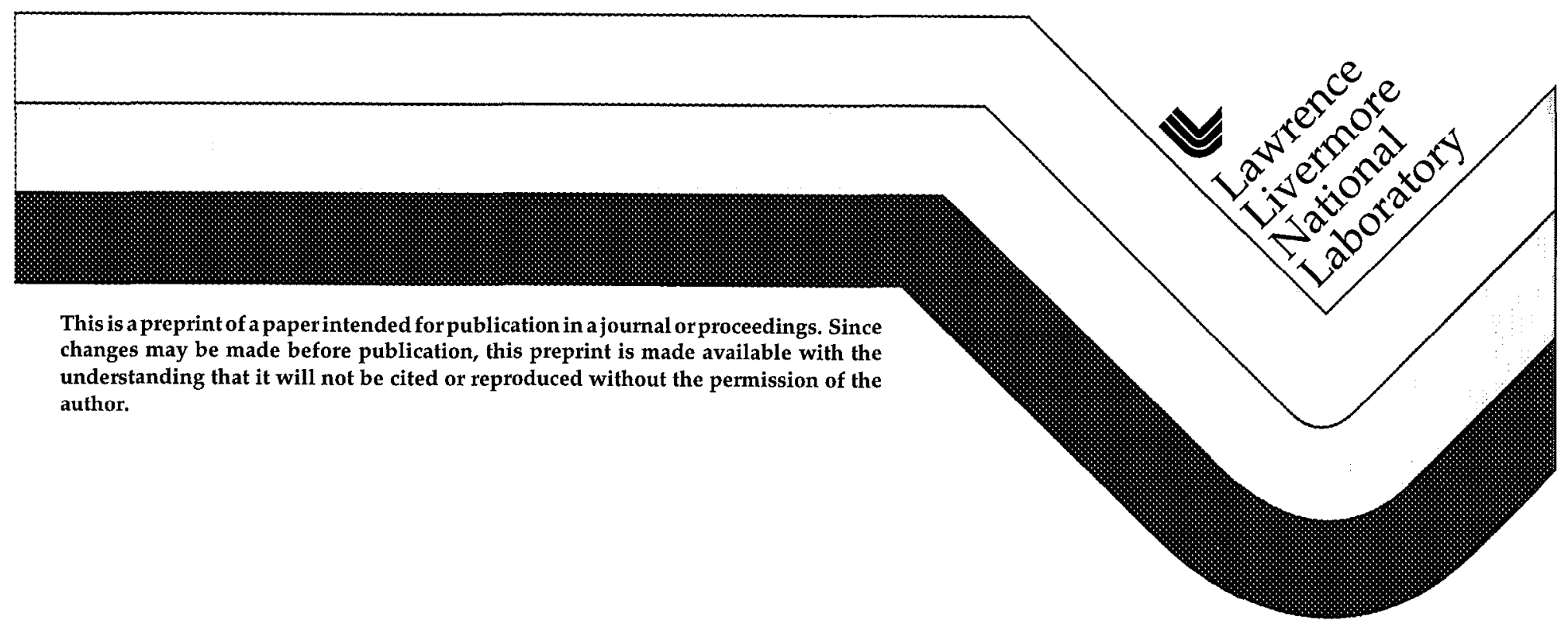




\section{DISCLAIMER}

This document was prepared as an account of work sponsored by an agency of the United States Government. Neither the United States Government nor the University of California nor any of their employees, makes any warranty, express or implied, or assumes any legal liability or responsibility for the accuracy, completeness, or usefulness of any information, apparatus, product, or process disclosed, or represents that its use would not infringe privately owned rights. Reference herein to any specific commercial product, process, or service by trade name, trademark, manufacturer, or otherwise, does not necessarily constitute or imply its endorsement, recommendation, or favoring by the United States Government or the University of California. The views and opinions of authors expressed herein do not necessarily state or reflect those of the United States Government or the University of California, and shall not be used for advertising or product endorsement purposes. 


\title{
Optical Assembly and Alignment for the National Ignition Facility Project
}

\author{
P. A. Hurst, E. L. Grasz, H. Wong, E. H. Schmitt, and M. R. Simmons \\ National Ignition Facility Operations Engineering Team \\ Lawrence Livermore National Laboratory, P.O. Box 808, L-447, Livermore, CA 94550
}

\begin{abstract}
The National Ignition Facility (NIF) will use about 8,000 large optics to carry a high-power laser through a stadium-size building, and will do so on a very tight schedule and budget. The collocated Optics Assembly Building (OAB) will assemble and align, in a clean-room environment, the NIF's large optics, which are the biggest optics ever assembled in such an environment. In addition, the OAB must allow for just-in-time processing and clean transfer to the areas where the optics will be used.

By using a mixture of off-the-shelf and newly designed equipment and by working with industry, we have developed innovative handling systems to perform the clean assembly and precise alignment required for the full variety of optics, as well as for postassembly inspection. We have also devcloped a set of loading mechanisms that safely get the clean optics to their places in the main NIF building.
\end{abstract}

\section{INTRODUCTION}

The National Ignition Facility Project is designing the National Ignition Facility (NIF), a multiapplication, stadium-size laser currently under construction at Lawrence Livermore National Laboratory (LLNL) for use primarily in the U.S. Department of Energy's Stockpile Stewardship Program. The NIF will be complete in 2003, and will use about 8,000 large optics of 26 different types (reflecting mirrors, neodymium-doped laser glass, etc.) to focus up to 192 laser beams on a dime-size target. The path of the laser will be completely contained within the huge Laser and Target Area Building (LTAB), but critical to the NIF's success will be the adjacent Optics Assembly Building (OAB).

The OAB is a separate facility whose role will be the assembly and alignment of the large NIF optics. ${ }^{1}$ The OAB will need to maintain rigorous cleanliness levels, achieve both the commonality and versatility to handle the various optic types, and deliver those optics into the LTAB without undoing their strict cleanliness and precise alignment. These requirements will apply not only during initial installation, but also during the NIF's lifetime operation, which also requires just-in-time processing. For the large optics, this is a considerable undertaking.

This will be the first time that such large optics will be assembled in a Class 100 clean-room environment. Some of the assemblies will weigh $3,000 \mathrm{lb}$, negating the utility of existing equipment (which can handle up to $20 \mathrm{lb}$ for such tasks). In addition, the Project's severe time and cost requirements place great demands on the precision alignment and transport of these optics. We have had to work closely with industry, using a mixture of new and off-the-shelf technology, new system designs, and custom modifications to fit our needs.

\subsection{Line-replaceable units}

We often refer to the optics as line-replaceable units (LRUs). The LRU reflects the Project's design philosophy of modularity. Should one optic of the 192-beam, 8,000-optic laser suffer damage, that optic will need to be removed and replaced quickly, safely, and cost-effectively. Optic LRUs are the packages for assembly, transportation, installation, and removal, and usually hold two or four separate optics. (In this paper, all LRUs discussed are optic LRUs.) 


\section{OAB FACILITY DESCRIPTION}

The OAB and LTAB are collocated to optimize the OAB's efficiency. As Figure 1 shows, the 25,000- $\mathrm{ft}^{2} \mathrm{OAB}$ comprises a loading dock, mechanical and optic transfer areas, a mechanical parts cleaning room, and an assembly and alignment area to manufacture, align, and test the LRUs. Both mechanical and optic components arrive at the OAB loading dock and undergo a receiving inspection in an enclosed area. After this inspection, they go into separate Class 10,000 unpackaging and preparation rooms; these transfer areas contain component handling mechanisms, cleaning stations, and a staging area.

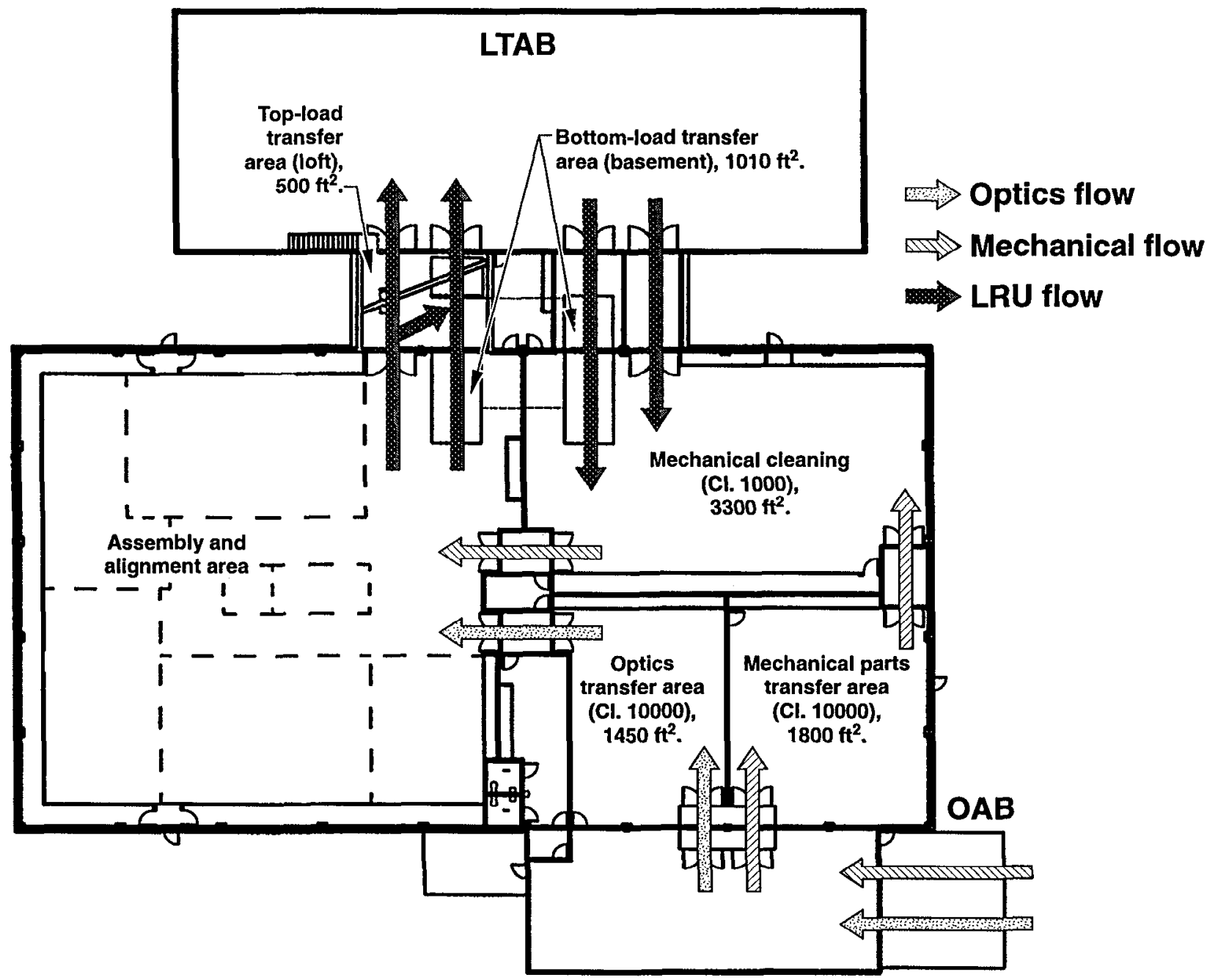

40-00-1297-2642pbo1

Figure 1. The NIF Optics Assembly Building will cleanly assemble and precision align all NIF large optics before delivering them to the Laser and Target Area Building.

All LRU mechanical parts are required to be cleaned to MIL-STD-1246C Level 50A or better prior to assembly (e.g., no more than $1665-\mu \mathrm{m}$ or $150-\mu \mathrm{m}$ particles/ $\left./ \mathrm{ft}^{2}\right)$. The precleaning systems consist of a self-contained, high-pressure spray with surfactant, if required. The precision cleaning system will use an ultrasonic bath with surfactant, rinse bath, high-pressure spray, and drying station. The optics arriving at the OAB have previously been processed and cleaned. 
From these transfer and cleaning areas, the components are distributed to various locations within the assembly and alignment clean room. This Class 100 clean room has five assembly areas, each of which is equipped with high-payload handling equipment (described in Section 3). In the middle of the assembly and alignment area is a final alignment and verification area (described in Section 4). From these separate components, the task at this point in the process is to assemble the LRUs for use in the LTAB.

Typically, an LRU includes a mechanical housing, laser optics (lenses and mirrors), utilities, and actuators. The optics are fragile and require delicate handling. The mechanical parts to be cleaned vary greatly in size, geometry, surface finish, and material, and range in size from machine screw parts to frame-like mechanical structures as long as nine feet. Materials are typically various grades of aluminum, steel, and stainless steel, each with a different surface finish. It is in this area that the engineering challenges truly hegin.

\section{ASSEMBLY AND ALIGNMENT}

The tasks to be performed in this area of the OAB are unprecedented. The Class 100 clean assembly of large optical assemblies is complicated by the fact that "off-the-shelf" equipment does not exist. Most modern clean rooms handle components and assemblies that weigh no more than $20 \mathrm{lb}$; the components in the $\mathrm{OAB}$ weigh from 150 to $3,000 \mathrm{lb}$, some the size and shape of a phone booth. Figure 2 shows examples of the various configurations of the LRUs and their weights. From a protocol perspective, we will achieve the cleanliness level by limiting the exposure of their individual components and the whole units to particle generators, and by providing delicate handling and maneuvering. To overcome these challenges, we are teaming with industry, leveraging designs that cleanly handle these large assemblies.

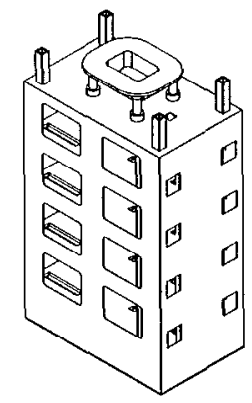

Spatial filter alignment tower, 3,000 lbs (top loaded)

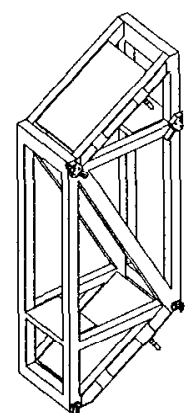

Periscope, $1,500 \mathrm{lbs}$ (bottom loaded)

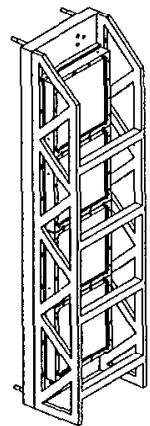

Spatial filter lens (bottom loaded)

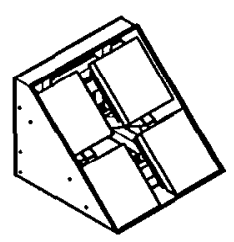

Laser mirror structure (switchyard)

Figure 2. Typical LRUs to be assembled, aligned, and transferred within the OAB.

\subsection{Rotating assembly table}

One key piece of equipment, known as a rotating assembly table, is a three-degree-of-freedom mechanism with one translational and two rotational axes. It is used to hold the LRU frames during their assembly. Prototype and test efforts are necessary to verify that the mechanisms are Class 100 clean room compatible and to assure that their load capacities are not exceeded. Figure 3 shows a rotating assembly table (left of photo) as part of the prototype assembly station.

\subsection{Optic insertion mechanism}

Another key piece of assembly hardware is the optic insertion mechanism (OIM). This manipulator installs large optic and mechanical components into LRU frames. The OIM is appropriately sized for the loading requirements of different components. Where possible, the OIM's manipulators are common end attachments (end effectors) that directly interface and handle the components. Figure 3 shows the OIM (right of photo) as part of the prototype assembly station. 


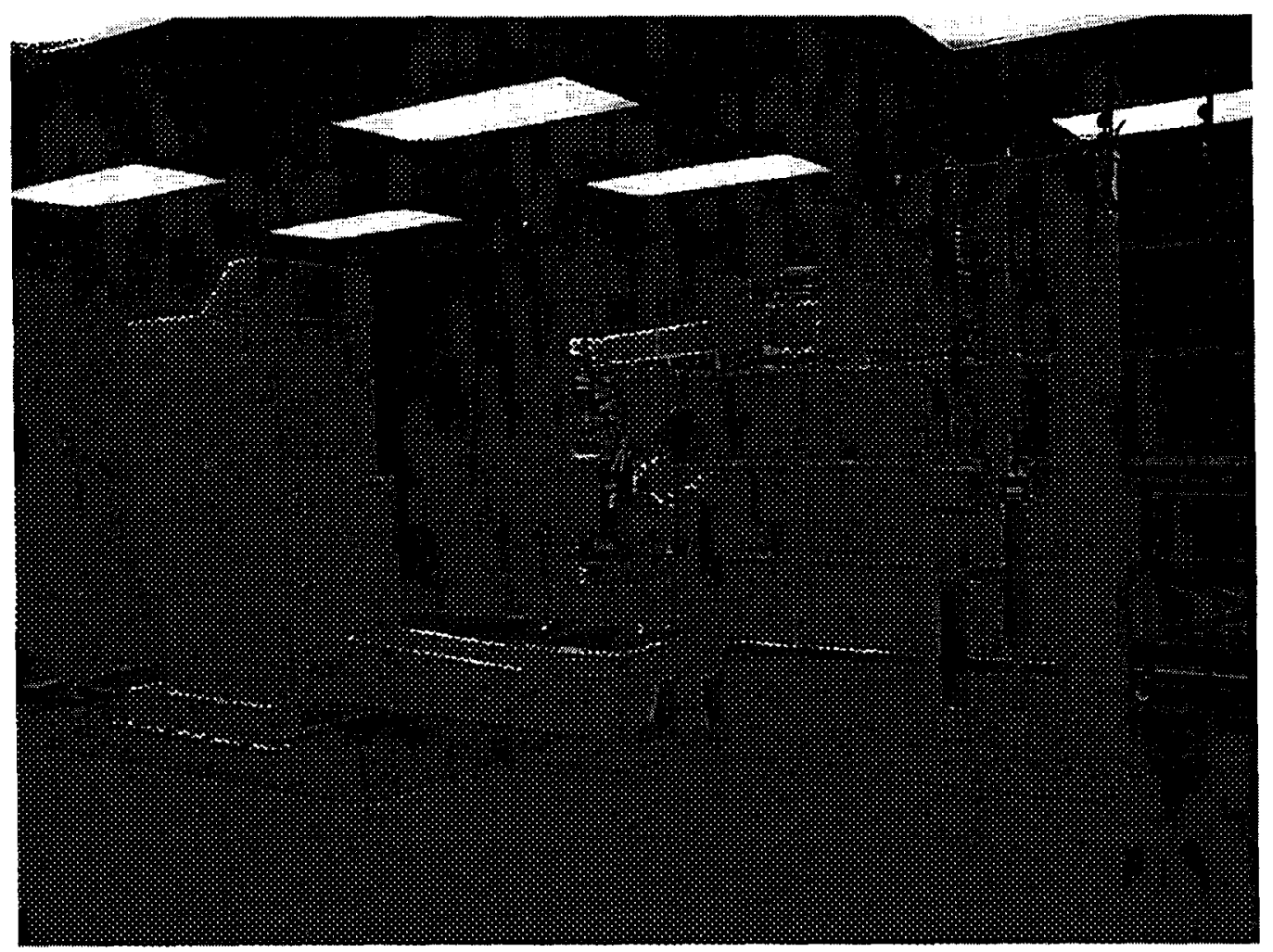

Figure 3. Prototype OAB assembly station, including rotating assembly table (left) and optic insertion mechanism (right).

The OIM is an electromechanical system allowing the operator to lift significant loads (up to $300 \mathrm{lb}$ ). Each assembly area in the OAB's overall assembly and alignment area will have an OIM. Due to the sensitivity of the optics and some mechanical components, we are designing and developing custom end effectors. The prototyping effort will both validate the mechanical operation and robustness and vcrify the OIM's cleanliness levels.

\subsection{OAB transport vehicle}

Transporting an assembled LRU within the OAB requires a common transport mechanism that functions with all of the specific LRUs. This is accomplished with the OAB's LRU transporter, shown in Figure 4. This transporter moves fully assembled LRUs to the next processing station within the OAB and to the transfer area for loading each LRU into its canister. With the maximum LRU loads of $3000 \mathrm{lb}$, and with lift height exceeding eight feet, the transporter must be able to both hold the load and distribute it across the OAB's raised floor. Prototyping will determine the proper handling methods and techniques for the completed LRU, and will test the lifting fixtures for function and cleanliness.

\section{INSPECTION AND VERIFICATION}

Before each LRU is transferred from the OAB into the LTAB for installation, its optics must be inspected for assemblyinduced stress. Traditionally, interferometry is used to qualify optics after mounting for use in lasers; this technique, however, is too expensive and vibration sensitive for the large volume and wide variety of the large-aperture optical systems to be handled in the $\mathrm{OAB}$. We are therefore developing a fast and inexpensive system to perform large-aperture interferometry for transmitted wavefront analysis and general polarization analysis and measurements of NIF optical components. In fact, this challenge is double-each optic is either reflective or transmissive, requiring two systems to complete this inspection step. We are aggressively pursuing two such systems, as well as a final cleanliness verification system for all of the LRUs. 


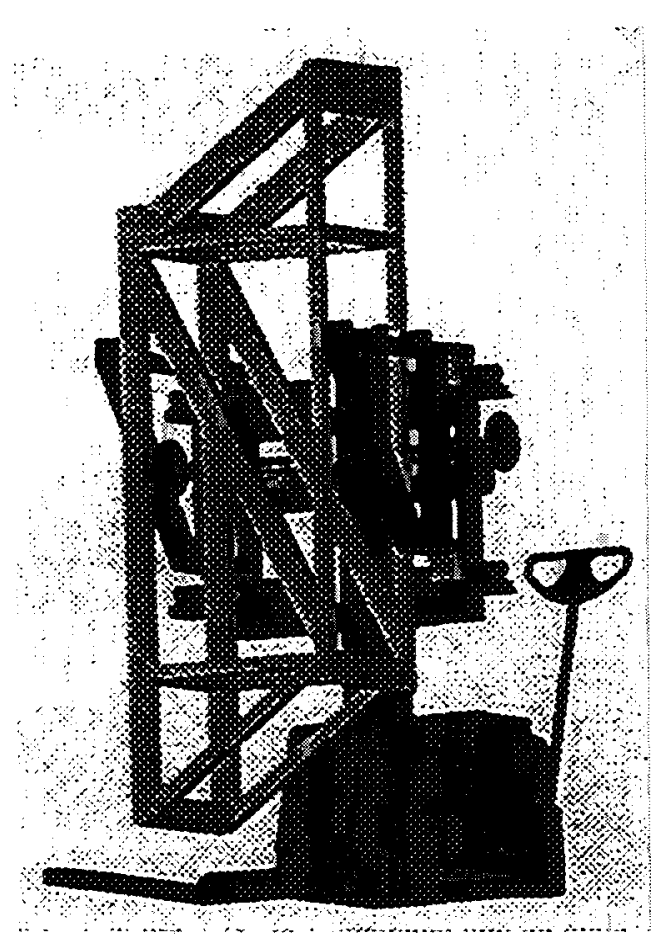

Figure 4. OAB LRU transporter carrying a "periscope" LRU.

\subsection{The profilometer: inspection of reflective optics}

For the reflective optics, NIF is currently involved in a proof-of-principle contract for development of a "profilometer." The profilometer is an optical profiler that uses multiple sensors in an innovative, self-referencing configuration. ${ }^{2}$ The sensors are mounted together on a rigid turntable, or platter. As we impart a compound rotary motion to the platter, we measure the proximity of the optical surface from each sensor. The result, after sophisticated mathematical analysis, is a map of the surface asphericity, which will indicate the presence and level of stress in the optics.

The map is independent of any small rigid-body motions of the sensor framework, and does not rely on any reference surface. This instrument has many advantages over conventional interferometers. It is relatively small, being comparable to the size of the test piece rather than being comparable to the radius of curvature. Also, it is equally applicable to convex and concave surfaces, and there is essentially no limitation on the speed (f-number) of the test piece. Finally, the testing orientation is arbitrary, which will allow for the required NIF testing at angles that range over $\pm 45^{\circ}$ from the horizontal. The standoff of the instrument from the test piece can be traded against total system accuracy. For example, with a standoff of a few millimeters, nanometer-class metrology appears feasible. For a standoff of 10 to $30 \mathrm{~cm}$, as would be appropriate for the NIF optics, accuracies better than one-tenth-wave should be routinely achievable.

\subsection{The polarimeter: inspection of transmissive optics}

The transmissive optics are inspected using a polarimeter. ${ }^{3}$ It has been demonstrated that variations in stress upon optical elements will cause variations in the polarization state of a transmitted wavefront. These improper polarization states result in loss in systems, which utilize Brewster amplifiers and frequency converters. The typical technique of measuring the birefringence is to place an optical element between two crossed polarizers to extinguish transmission and to determine if light transmitted is due to stress or intrinsic birefringence. It has been demonstrated that by rotating an analyzer between two 
crossed polarizers, the transmission varies sinusoidally as the analyzer is rotated in phase. A slight modification to conventional phase-modulating interferometry software used on commercial phase-shifting interferometers can yield the phase retardance in transmission instead of wavefront. This phase retardance is mapped over the full aperture of an optical system, essentially drawing a picture in which the lines easily show stress in the optic. Note that the polarimeter will be used as a "pass-fail" test for assembly, not as an analytical evaluation of the optical surfaces (they are tested thus before entering the OAB).

\subsection{Cleanliness verification}

The final inspection step for the LRUs is the cleanliness verification station. Assembled LRUs are evaluated for particle contaminants $5 \mu \mathrm{m}$ and larger. We are in the process of evaluating the current technologies being used at this time in the industry to determine whether they can be used to collect the cleanliness data. Currently, inspection camera systems are being used to evaluate bulk defects in laser glass. The configuration is a telecentric laser scanner and fully focused collector, operating in both bright field and dark field. This sensor will provide the requisite detection and will be mountable on a moving bridge or robot arm, in a limited-installation envelope.

Early evaluation of this system has yielded results potentially applicable to surface contamination evaluations. In addition, high-throughput laser scanners are being used in the float glass industry and may be applicable to NIF LRU surface-particle contamination evaluation. The current defect size in the float glass industry is not as stringent as NIF requirements.

\section{LRU LOADING AND TRANSFER}

Assembling clean LRUs is useless, of course, if they get dirty on the way to the LTAB. From the OAB, each LRU will either be placed through an airlock onto a forklift or will be loaded into a clean canister that will be carried by the LTAB transport vehicle. From here, the LRU will be carried to its proper place in the LTAB. This route will be reversed when an LRU needs to be replaced or repaired. We have designed the transfer mechanism so that the interface between the OAB and the transfer vehicle is identical (or nearly identical) to the interface between the transfer vehicle and the LTAB.

With so many different LRU designs, we have had to design three different mechanisms for transferring the LRU out of the OAB: direct airlock transfer to a forklift, top-loading into a clean canister, and bottom-loading into a clean canister. The two canister mechanisms include a special transfer vehicle, as well as a cover-removal mechanism that serves as a barrier between the OAB and the dirty basement and loft. The loft and basement allow the vehicles to perform top- and bottom-loading (from either above or below the OAB's main floor, respectively).

\section{CONCLUSION}

The NIF will use a tremendous variety of optics to send a high-power laser through the stadium-size LTAB, and will do so on a very tight schedule and budget. The $\mathrm{OAB}$ will assemble and align, in a clean-room environment, the NIF's large optics, which are the biggest optics ever assembled in such an environment. In addition, the OAB must allow for just-in-time processing and clean transfer to the areas where the optics will be used.

The combination of commonality and versatility required for this task creates a daunting set of engineering and design challenges. Using and adapting off-the-shelf equipment and working with industry, we have developed such tools as the rotating assembly table and the optic insertion mechanism for precise assembly and alignment, as well as the profilometer and polarimeter for postassembly inspection. A set of loading mechanisms gets the clean optics to an LTAB transport vehicle, which carries it to its place in the LTAB. 


\section{ACKNOWLEDGMENTS}

The design of the $\mathrm{OAB}$, the material handling designs, and the results noted in this paper have been prepared with the support of the Optical LRU Assembly and Alignment Design Group within the NIF Operations Engineering Section. This dedicated team included Leslie Allison, Rudy Carpenter, Don Fleming, Brett Hall, Alan Havassy, Ray Iaea, Sudair Jain, Bob Koczian, Kent Lueng, Mike McDaniel, Roy Merrill, Ken Montgomery, Sandra Owens, Mark Perez, Tamra Porter, Jo Sander, Dennis Silva, Kay Sivori, Ladonna Willis, and Marty Yeoman.

This work is performed under the auspices of the U.S. Department of Energy by Lawrence Livermore National Laboratory under Contract W-7405-ENG-48.

\section{REFERENCES}

1. Title II Planning Document for NIF Operations Engineering, WBS 1.9, NIF-0002560 (June 1997).

2. Proposal for a Proof of Concept for a Large-Area Surface-Figure-Mapping Instrument for NIF Optics, RFP \#P342504.

3. Imaging Polarimeter for Birefringence/Stress Analysis of NIF Components; Paul Wilkens, LLNL (April 1997). 


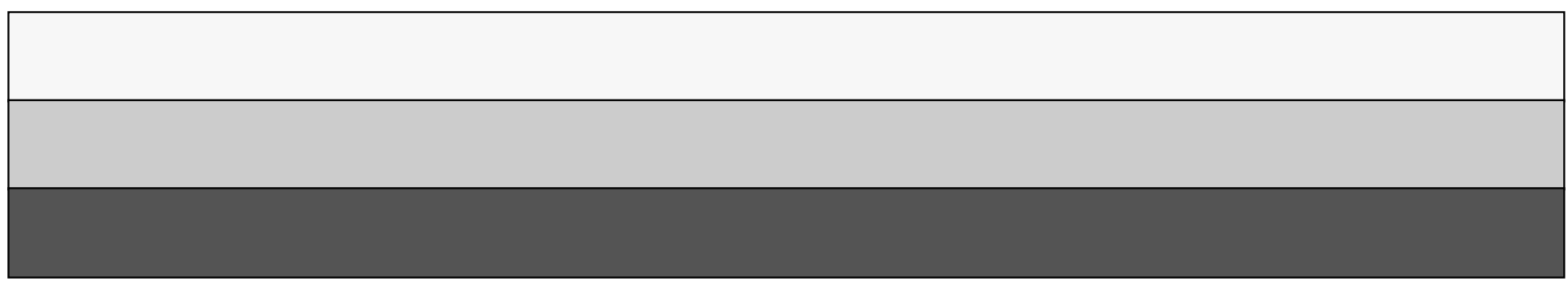

